\title{
Studying local order in ferroelectric oxide superlattices with three-dimensional diffuse X-ray scattering
}

\author{
Joohee Bang ${ }^{1}$, Nives Strkalj ${ }^{1,2}$, Martin Sarott ${ }^{1}$, Morgan Trassin ${ }^{1}$, Manfred Fiebig ${ }^{1}$, Thomas Weber ${ }^{1}$ \\ ${ }^{1}$ Department of Materials Science, ETH Zurich, Switzerland \\ ${ }^{2}$ Department of Materials Science and Metallurgy, Cambridge University, United Kingdom
}

joohee.bang@mat.ethz.ch

Thin film structures that exhibit exotic phases and topologies have attracted the attention of diverse condensed matter communities. Xray characterization-based investigations on such systems have been so far restricted to the study of microstructural analysis such as epitaxial match between substrate and film and of the polarization domain sizes and arrangements. Detailed investigations of the local atomic structure and the complex interplay between polarization states within the domains and the domain walls have remained elusive with X-ray diffraction. Here, we present an approach for atomic-scale structural characterization of ferroelectric single crystalline thin films based on comprehensive three-dimensional diffuse scattering data sets. The diffuse scattering will be evaluated with the three-dimensional pair distribution function (3D- $\mathrm{PDDF}$ ) method ${ }^{1}$. Specifically, we investigate superlattices of alternating ferroelectric lead titanate and dielectric strontium titanate layers with complex electrical polarization structures ${ }^{2,3}$. This work does not only gain insights on structure-property correlations of epitaxially grown single crystalline thin films, but also lay groundwork for developing experimental and modelling tools for analyzing the local structures of thin films.

[1] Weber \& Simonov 2012

[2] Yadav A. et al. 2016

[3] A. R. Damodaran et al. 2017

Keywords: diffuse scattering, three-dimensional delta pair distribution function, X-ray characterization, ferroelectric thin films, polarization domains 